\title{
Use of electronic brachytherapy to deliver postsurgical adjuvant radiation therapy for endometrial cancer: a retrospective multicenter study
}

\author{
This article was published in the following Dove Press journal: \\ OncoTargets and Therapy \\ 17 September 2010 \\ Number of times this article has been viewed
}

\author{
William C Dooley' \\ John P Thropay ${ }^{2}$ \\ Gary J Schreiber ${ }^{3}$ \\ Mohamed Y Puthawala ${ }^{4}$ \\ Steven C Lane ${ }^{5}$ \\ James CWurzer 6 \\ Charles E Stewart ${ }^{7}$ \\ Gordon L Grado ${ }^{8}$ \\ Harish G Ahuja9 \\ Gary M Proulx ${ }^{10}$
}

'University of Oklahoma Health Sciences Center, Oklahoma City, OK; ${ }^{2}$ Beverly Oncology and Imaging Center, Montebello, CA; ${ }^{3}$ Swedish Covenant Medical Center, Chicago, IL; ${ }^{4}$ Rhode Island Hospital, Providence, RI; ${ }^{5}$ Signature Healthcare Brockton Hospital, Brockton, MA; ${ }^{6}$ AtlantiCare Regional Medical Center, Egg Harbor Township, NJ; ' ${ }^{7}$ t Francis Medical Center, Tulsa, OK; ${ }^{8}$ Southwest Oncology Centers, Scottsdale, AZ; ${ }^{9}$ Aspirus Regional Medical Center, Wausau, WI; ${ }^{10}$ Exeter Hospital, Exeter, $\mathrm{NH}$, USA

Correspondence: William C Dooley The University Oklahoma Health Sciences Center, Oklahoma City, OK, 73I04, USA

Tel +| $40527 \mid 7867$

Fax + I 405 27| 4443

Email william-dooley@ouhsc.edu

\begin{abstract}
Background: This retrospective, multicenter study evaluated the feasibility and safety of high-dose rate electronic brachytherapy (EBT) as a postsurgical adjuvant radiation therapy for endometrial cancer.

Methods: Medical records were reviewed from 41 patients (age 40-89 years) with endometrial cancer (Federation of International Gynecology and Obstetrics stages IA-IIIC) treated at nine centers between April 2008 and October 2009. Treatment included intracavitary vaginal EBT alone $(n=16)$ at doses of 18.0-24.0 Gy in 3-4 fractions and EBT in combination with external beam radiation therapy (EBRT, $\mathrm{n}=25$ ) at a total radiation dose range of 40.0-80.4 Gy. Doses were prescribed to a depth of $5 \mathrm{~mm}$ from the applicator surface and to the upper third $(\mathrm{n}=15)$ and the upper half $(n=26)$ of the vagina.
\end{abstract}

Results: Median follow-up was 3.8 (range 0.5-12.0) months. All 41 patients received the intended dose of radiation as prescribed. Adverse events occurred in 13 of 41 patients and were mild to moderate (Grade 1-2), consisting primarily of vaginal mucositis, rectal mucosal irritation and discomfort, and temporary dysuria and diarrhea. There were no Grade 3 adverse events in the EBT-only treatment group. One patient, who was being treated with the combination of EBT and EBRT for recurrent endometrial cancer, had a Grade 3 adverse event. No recurrences have been reported to date.

Conclusion: Electronic brachytherapy provides a feasible treatment option for postoperative adjuvant vaginal brachytherapy as sole radiation therapy and in combination with EBRT for primary endometrial cancer. Early and late toxicities were mild to moderate.

Keywords: endometrial cancer, electronic brachytherapy, radiation therapy

\section{Introduction}

Endometrial cancer is the most common gynecologic cancer and the fourth most common type of cancer in women in the Western world. ${ }^{1}$ An estimated 42,160 women were diagnosed with endometrial cancer in 2009 in the US, ${ }^{1}$ with a majority of cases having early-stage disease. ${ }^{2}$ Early-stage endometrial cancer has a good prognosis, and five-year survival rates have recently increased to $80-90 \%$ in women who were treated with total abdominal hysterectomy, bilateral salpingo-oophorectomy (TAH-BSO), and adjuvant radiation therapy for Stage I disease. In a survey of over 21,000 patients with Stage I endometrial cancer, adjuvant radiation therapy was found to be significantly associated with improved survival. ${ }^{3}$ However, external beam radiation therapy (EBRT) in early-stage endometrial cancer has become less common due to the time and 
morbidity associated with this form of low-dose rate radiation. High-dose rate (HDR) intracavitary brachytherapy, which provides dose rates greater than 100 centigray per hour, can be completed in a shorter time on an outpatient basis, with decreased radiation exposure to nontarget organs and tissue. With the increased trend to stage endometrial cancer patients surgically, vaginal brachytherapy as sole radiation therapy or combined with other modalities has become an essential part of adjuvant treatment for endometrial cancer. Numerous studies of the use of vaginal brachytherapy have demonstrated good control rates with minimal morbidity. ${ }^{4-13}$

HDR vaginal brachytherapy has typically relied on an ${ }^{192}$ iridium source. However, this form of radiation requires an HDR afterloader unit and a shielded radiation vault, which is not financially feasible in smaller institutions or clinics and can present scheduling challenges at higher-volume centers. Electronic brachytherapy (EBT) utilizes a miniaturized 50 kilovoltage $(\mathrm{kV}) \mathrm{X}$-ray source that does not require a vault or an HDR afterloader unit. Minimal shielding, in the form of a rolling shield for staff and a half-apron over the lower abdomen of the patient, allows the therapist to be present in the treatment room, which significantly increases patient comfort. The device, once state registration, health physics, and regulatory requirements are met, can be moved from one procedure room to the next. EBT does not require storage and handling of isotopes. ${ }^{14,15}$

The Axxent ${ }^{\circledR}$ EBT system (Xoft, Inc, Sunnyvale, CA) has been utilized in the US for the treatment of breast cancer since 2005. ${ }^{15}$ In 2008, the US Food and Drug Administration provided clearance for the use of this EBT system with specifically designed applicators for the treatment of endometrial cancer. The objective of this retrospective multicenter study was to assess treatment feasibility and acute adverse events as documented in the records of patients treated with EBT as an adjuvant therapy for endometrial cancer.

\section{Methods}

Medical records were reviewed from 41 patients at nine institutions (Beverly Oncology and Imaging Center, Montebello, CA; Swedish Covenant Medical Center, Chicago, IL; Rhode Island Hospital, Providence, RI; Signature Healthcare Brockton Hospital, Brockton, MA; AtlantiCare Regional Medical Center, Egg Harbor Township, NJ; St Francis Medical Center, Tulsa, OK; Southwest Oncology Centers, Scottsdale, AZ; Aspirus Regional Medical Center, Wausau, WI; and Exeter Hospital, Exeter, NH. The protocol was approved by the institutional review boards at the nine participating centers.

\section{Patients}

The medical records were reviewed for 41 patients with endometrial cancer who were treated with vaginal EBT between April 2008 and October 2009; follow-up visits took place between November 2008 and December 2009. Records of patients treated with EBT alone or EBT in combination with EBRT were included. Records of patients currently enrolled in any other EBT study were excluded. No other exclusion criteria were established. The staging of endometrial cancer for each patient was done according to the Federation of International Gynecology and Obstetrics (FIGO) staging criteria (1988). The histopathologic grade was defined as G1 (well differentiated), G2 (moderately differentiated), and G3 (poorly differentiated).

\section{Data collection}

The data were collected retrospectively, with the centers sequentially numbered, and with patients sequentially numbered using three-digit numbers within each center. Adverse events were collected using Common Terminology Criteria for Adverse Events version 3.0. Adverse events were rated based on the Common Terminology Criteria for Adverse Events version 3, and included grades $1=$ mild, $2=$ moderate, 3 = severe/undesirable, 4 = life-threatening/ disabling, $5=$ death related to adverse event.

\section{Materials}

Vaginal EBT was delivered using the Axxent EBT system. The EBT system consists of the X-ray source, the vaginal cylinder applicator, and the controller. The X-ray source comprises a miniaturized $50 \mathrm{kV}$ X-ray tube in a multilumen catheter that allows cooling fluid to circulate over the tube. The vaginal cylinder applicators were designed to provide transmission characteristics specifically for the low energy X-rays emitted by the electronic X-ray source. The cylinders are composed of common medical-grade polymers, and have a 94\% $\pm 5 \%$ $\mathrm{X}$-ray transmission with respect to water. The X-ray tube is approximately $2.25 \mathrm{~mm}$ in diameter and $15 \mathrm{~mm}$ long, attached to a high-voltage cable, and encapsulated within an electrical ground. The controller provides power to the X-ray source and allows the X-ray source to be translated within the applicator. The translation or pullback movement of the X-ray source within the applicator is designed to provide a desired dose of radiation in the tissue surrounding the cylinder.

\section{Treatment}

The prescription dose and brachytherapy treatment plans were prepared individually for each patient, typically based 
on CT scans. BrachyVision ${ }^{\mathrm{TM}}$ treatment planning software (Varian Medical Systems, Palo Alto, CA) or Plato ${ }^{\text {TM }}$ treatment planning software (Nucletron, Columbia, MD) were used at most centers. A vaginal cylinder applicator was selected for each patient from the four sizes available $(20 \mathrm{~mm}, 25 \mathrm{~mm}$, $30 \mathrm{~mm}, 35 \mathrm{~mm}$ ). The applicator was inserted just prior to treatment and removed following treatment on each treatment visit. Follow-up visits occurred periodically based on the standard practice at each study site.

\section{Statistics}

Case report forms were submitted to a data coordinating center where data were entered into a Access ${ }^{\circledR}$ (Microsoft, Redmond, WA) database by an independent data manager. Analysis of the data was performed using SAS statistical analysis software (Version 9.1.3; Sas Institute, Cary, NC). The number of observations (n) and proportion are reported for both the treatment success and acute outcome endpoints.

\section{Results \\ Patients}

Records from 41 female patients were reviewed, most of whom (93\%) were aged 50 years or older, with $31 \%$ being aged 70 years or older. Patient characteristics are listed in Table 1. A majority (68\%) had early-stage endometrial cancer (stages IA-IIA) according to the FIGO gynecologic staging system (1988). The remaining patients had cancer stages IIB-IIIC, including one patient with recurrent cancer. The median follow-up was 3.8 (range 0.5-12.0) months.

\section{Treatment}

Patients with primary endometrial cancer $(\mathrm{n}=40)$ and recurrent endometrial cancer $(n=1)$ were treated with vaginal EBT alone $(n=16)$ or EBT in combination with EBRT $(n=25)$. All 41 patients received the intended dose of radiation as prescribed. When EBT was used alone, the mean prescription dose was 21.3 Gy (standard deviation, $\mathrm{SD}=1.2 \mathrm{~Gy}$ ) and was independent of FIGO stage as shown in Table 2. When EBT was combined with EBRT $(n=24)$, excluding the patient treated for recurrence, the total radiation dose of the two therapies combined ranged from 40 Gy to 70.4 Gy with a mean value of $60.7 \mathrm{~Gy}(\mathrm{SD}=5.8 \mathrm{~Gy})$. Again, the total dose was independent of FIGO stage for these patients as indicated in Table 3. EBT dose was prescribed to a depth of $0.5 \mathrm{~cm}$ from the surface of the applicator, and the upper third to the upper half of the vagina was treated. The most commonly used vaginal cylinder diameter was $30 \mathrm{~mm}$ from a range of $20 \mathrm{~mm}$ to $35 \mathrm{~mm}$ (Table 4).
Table I Patient characteristics

\begin{tabular}{|c|c|}
\hline & n (\%) \\
\hline Patients (n) & $41(100 \%)$ \\
\hline \multicolumn{2}{|l|}{ Age range (years) } \\
\hline $40-49$ & $3(7.3 \%)$ \\
\hline $50-59$ & $12(29.3 \%)$ \\
\hline $60-69$ & $13(31.7 \%)$ \\
\hline 70-79 & $10(24.4 \%)$ \\
\hline $80-89$ & $3(7.3 \%)$ \\
\hline \multicolumn{2}{|l|}{ FIGO cancer stage ${ }^{a}$} \\
\hline IAG unspecified & I (2.4\%) \\
\hline IBG I & $3(7.3 \%)$ \\
\hline IBG2 & $4(9.8 \%)$ \\
\hline IBG3 & $8(19.5 \%)$ \\
\hline IBG unspecified & I (2.4\%) \\
\hline ICGI & $2(4.9 \%)$ \\
\hline ICG2 & $3(7.3 \%)$ \\
\hline ICG3 & I (2.4\%) \\
\hline ICG unspecified & $2(4.9 \%)$ \\
\hline IIAGI & I (2.4\%) \\
\hline IIAG2 & I (2.4\%) \\
\hline IIAG3 & I (2.4\%) \\
\hline IIBG I & I (2.4\%) \\
\hline IIBG2 & $2(4.9 \%)$ \\
\hline IIBG3 & $2(4.9 \%)$ \\
\hline IIIAG3 & $2(4.9 \%)$ \\
\hline IIIAG unspecified & I (2.4\%) \\
\hline IIICG2 & $2(4.9 \%)$ \\
\hline IIICG $3^{b}$ & $3(7.3 \%)$ \\
\hline Unspecified (high-grade) ${ }^{c}$ & I $(2.4 \%)^{d}$ \\
\hline \multicolumn{2}{|l|}{ Grading } \\
\hline GI & $7(17.1 \%)$ \\
\hline G2 & $12(29.3 \%)$ \\
\hline G3 & $16(39 \%)$ \\
\hline G unspecified & $6(14.6 \%)$ \\
\hline \multicolumn{2}{|c|}{ Time (days) from hysterectomy to first $E B T^{e}$} \\
\hline Mean \pm SD & $96.2 \pm 60.1$ \\
\hline Median & 80.0 \\
\hline Range & $20-255$ \\
\hline
\end{tabular}

Notes: a Federation International Gynecology and Obstetrics (FIGO) gynecologic cancer staging system; bone patient in this group was treated for recurrent endometrial cancer; "the "unspecified (high-grade)" cancer was a primary endometrial cancer; 'due to rounding, numbers do not total exactly $100 \%$; eincludes only patients with primary endometrial cancer in this study.

Abbreviations: EBT, electronic brachytherapy, SD, standard deviation.

\section{Adverse events}

Of 41 patients, $13(31.7 \%)$ had adverse events. Adverse events were mild to moderate (Grade 1-2) in 12 of the 13 patients, and there was one occurrence of a Grade 3 adverse event in one patient. Adverse events consisted primarily of temporary diarrhea and dysuria, vaginal mucositis, and rectal mucosal irritation and discomfort. The majority of patients $(68.3 \%)$ experienced no gastrointestinal or genitourinary side effects. All adverse events are listed in Table 5, and the severity grade is shown if it was recorded in the medical record. 
Table 2 Summary of prescription doses for patients receiving electronic brachytherapy only categorized by FIGO stage

\begin{tabular}{lllll}
\hline $\begin{array}{l}\text { FIGO } \\
\text { stage }\end{array}$ & Patients (n) & $\begin{array}{l}\text { EBT dose } \\
(\mathbf{G y})\end{array}$ & $\begin{array}{l}\text { Fractions } \\
(\mathbf{n})\end{array}$ & $\begin{array}{l}\text { Mean total } \\
\text { dose (Gy) }\end{array}$ \\
\hline IA & - & - & - & - \\
IB & 9 & $18-24$ & 3,4 & 21.3 \\
IC & 4 & $21-22$ & 3,4 & 21.3 \\
IIA & 2 & 21 & 3 & 21 \\
IIB & - & - & - & - \\
IIIA & I & 22 & 4 & 22 \\
IIIC & - & - & - & - \\
Unknown & - & - & - & - \\
Total & I6 & - & - & $21.3 \pm 1.2$
\end{tabular}

Abbreviations: Gy, gray; EBT, electronic brachytherapy; FIGO, Federation International Gynecology and Obstetrics.

Of the 16 patients treated with EBT alone 12 (75.0\%) were followed for more than three months and six (37.5\%) for more than six months. There were no early adverse events occurring in the first three months post-treatment in these patients. Late adverse events first reported at least three months after the last EBT fraction included four adverse events in three patients. One patient with blood tinged stool (day 119) and rectal bleeding (day 210) was diagnosed with a superficial rectal ulcer with Grade 2 toxicity during colonoscopy; the last follow-up visit (day 328) indicated that the events were less frequent with no alteration of gastrointestinal function. One patient (day 125) had anal discomfort, which resolved completely, and discomfort with intercourse, which did not require intervention. One patient had Grade 1 vaginal mucositis at day 115, and subsequent follow-up has not yet occurred.

The single recorded Grade 3 adverse event in the study was vaginal mucosa toxicity, with chronic moist desquamation recorded in the medical record at day 79 following the last brachytherapy fraction. No further mention of this finding was found in this patient's medical record at the next follow-up visit (day 128). At that point, the record did show vaginal discomfort, irritation, and occasional serosanguinous discharge following the use of a dilator. This patient was the one patient in the study with recurrent endometrial cancer. The primary cancer was diagnosed in mid-2005 with poorly differentiated Stage III endometrial carcinoma and treated with TAH-BSO, segmental colonic resection, and six cycles of chemotherapy (carboplatin and paclitaxel). The cancer recurred with distant metastases and friable exophytic vaginal lesions from the previously diagnosed endometrial cancer. She received an additional six cycles of chemotherapy (carboplatin and gemcitabine) prior to the study treatment, which was a combined radiation treatment with 55.4 Gy EBRT and 25 Gy EBT, administered in five fractions using a $30 \mathrm{~mm}$ applicator.

\section{Discussion}

This retrospective multicenter study evaluated treatment outcomes in patients treated with vaginal EBT as postsurgical adjuvant therapy for endometrial cancer. The study included patients with stages IA-IIIC endometrial cancer, according to the FIGO gynecologic staging system, and included one patient with recurrent Stage IIIC endometrial cancer. As a retrospective study, there is greater variation in the treatment protocols between patients than would have been treated in a prospective study with specific patient selection criteria and a treatment protocol. In this study, the prescribed dose range of vaginal EBT ranged from 8.0 to $30.0 \mathrm{~Gy}$, and was successfully delivered as prescribed in all 41 patients. The treated area of

Table 3 Summary of prescription doses for patients receiving electronic brachytherapy plus external beam radiation therapy categorized by FIGO stage

\begin{tabular}{|c|c|c|c|c|c|c|}
\hline FIGO stage & Patients $(n=24)$ & EBT dose (Gy) & Fractions (n) & EBRT dose (Gy) & $\begin{array}{l}\text { Combined dose } \\
\text { range (Gy) }\end{array}$ & $\begin{array}{l}\text { Mean combinec } \\
\text { dose (Gy) }\end{array}$ \\
\hline IA & 1 & 20 & 4 & 50.4 & 70.4 & 70.4 \\
\hline IB & 7 & $|2-2|$ & 3,4 & $20-50.4$ & $40-66$ & 58.5 \\
\hline IC & 4 & $10-18$ & $2,3,4$ & $45-59.4$ & $6 I-69.4$ & 64.1 \\
\hline IIA & I & 15 & 3 & 45 & 60 & 60.0 \\
\hline IIB & 5 & $|2-2|$ & 3 & $45-46.8$ & $57-66$ & 60.7 \\
\hline IIIA & I & 20 & 5 & 45 & 65 & 65.0 \\
\hline IIIC & 4 & $8-18$ & 2,3 & $41.4-50.4$ & $57-60$ & 58.7 \\
\hline $\begin{array}{l}\text { Unknown } \\
\text { (high-grade) }\end{array}$ & 1 & 12 & 2 & 44 & 56 & 56 \\
\hline Mean $\pm S^{a}$ & & $15.3 \pm 3.7$ & & $45.4 \pm 6.4$ & & $60.7 \pm 5.8$ \\
\hline Recurrence IIIC & I & 25 & 5 & 55.4 & 80.4 & 80.4 \\
\hline
\end{tabular}

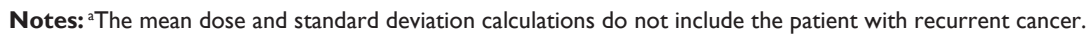

Abbreviations: Gy, gray; EBRT, external beam radiation therapy; EBT, electronic brachytherapy; SD, standard deviation; FIGO, Federation International Gynecology and Obstetrics. 
Table 4 Electronic brachytherapy treatment summary

\begin{tabular}{ll}
\hline Treated area & $\mathbf{n}(\%)$ \\
\hline Upper half & $26(63)$ \\
Upper third & $15(37)$ \\
Applicator size (diameter) & \\
$20 \mathrm{~mm}$ & $6(14)$ \\
$25 \mathrm{~mm}$ & $8(20)$ \\
$30 \mathrm{~mm}$ & $24(59)$ \\
$35 \mathrm{~mm}$ & $3(7)$ \\
\hline
\end{tabular}

the upper third to upper half of the vagina, treatment depth, and selected cylinder diameters are consistent with reported practice patterns. ${ }^{16}$ Treatment times were not collected in this study, but would be expected to be consistent with the mean treatment time of 4.9 minutes reported by Dickler et al in a prospective study of 15 patients with endometrial cancer treated with mean EBT doses of $20.2 \mathrm{~Gy}$, which is comparable with our mean dose of 20.0 Gy. ${ }^{15}$

No recurrences have been reported to date in these patients; however, the duration of follow-up is less than one year in nearly all patients. Numerous studies of the use of vaginal brachytherapy have demonstrated good control rates with minimal morbidity. ${ }^{4-13}$ Recently, a large multicenter study showed that vaginal brachytherapy treatment alone compared with EBRT provides a better quality of life over EBRT and should be the preferred treatment from a quality of life perspective, when appropriate, as the sole treatment for endometrial cancer. ${ }^{17}$

In this study, the majority of patients (28/41) had no adverse events, and 12 patients, who were treated for primary endometrial cancer, had only low-grade adverse events (Grade 1 or 2) following treatment. These results are consistent with those reported by Dickler et al in which 15 patients were treated with EBT alone or in combination with EBRT for endometrial cancer. ${ }^{18}$ In the present study, one patient, who was treated for a recurrence in the combined radiation group, exhibited a Grade 3 adverse event. Adverse events were numerically more common in the combined radiation group (36.0\%) compared with the EBT alone group (18.8\%); however, the small sample size does not warrant statistical testing. Interestingly, the EBT alone group had no acute adverse events during the first three months following the last fraction. The four events that occurred in this group were first recorded at 3.8 months post-treatment or later (Table 5). In contrast, 16 of 20 events that occurred

Table 5 Adverse events by patient following treatment with electronic brachytherapy alone or in combination with external beam radiation therapy as recorded at follow-up visits

\begin{tabular}{|c|c|c|c|}
\hline Patient number & AEs for patients treated with EBT and EBRT & Days post EBT $(n)$ & CTC Grade \\
\hline $1-1$ & Diarrhea & 5 & 1 \\
\hline $1-2$ & Diarrhea & 10 & I \\
\hline $2-1$ & Diarrhea, fatigue & 43 & $\mathrm{I}, 2$ \\
\hline $3-1$ & Erythema at introitus & 14 & 1 \\
\hline $3-2$ & Diarrhea & 36 & I \\
\hline \multirow[t]{2}{*}{$4-1$} & Dysuria, abdominal discomfort & 2 & 1 \\
\hline & Hemorrhoid, abdominal discomfort & 99 & 2,1 \\
\hline \multirow[t]{4}{*}{$4-2$} & Dysuria related to external beam therapy & 14 & 2 \\
\hline & Fungal skin rash related to external beam therapy & 14 & 2 \\
\hline & Burning/flushing sensation at tumor site related to external beam & 13 & 2 \\
\hline & Diarrhea related to external beam therapy & 13,111 & I, 2 \\
\hline \multirow[t]{5}{*}{$4-3$} & Dysuria & 13 & 2 \\
\hline & Diverticulosis, hepatomegaly related to EBT & 111 & $\mathrm{I}, \mathrm{I}$ \\
\hline & Renal stone (small right) & 111 & NA \\
\hline & Dysuria (end micturition) & 38,79 & NA \\
\hline & Bladder spasms, occasional & 38,79 & NA \\
\hline \multirow[t]{3}{*}{$4-4$} & Chronic moist desquamation of vaginal mucosa & 79 & 3 \\
\hline & Vaginal discomfort/irritation & 38,128 & NA \\
\hline & Serosanguinous discharge with use of dilator (occasional) & 128 & NA \\
\hline $4-5$ & Urgency (occasional) & 85 & NA \\
\hline \multicolumn{4}{|c|}{ AEs for patients treated with EBT only } \\
\hline \multirow[t]{2}{*}{$5-1$} & Blood-tinged mucous stool & 119 & NA \\
\hline & Superficial rectal ulcer with bleeding & $210,266,328$ & 2 \\
\hline $6-1$ & Anal discomfort, discomfort with intercourse & 125 & NA \\
\hline $7-1$ & Vaginal mucositis & 115 & 1 \\
\hline
\end{tabular}

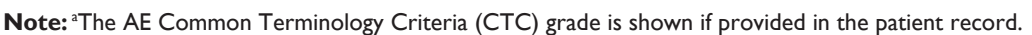

Abbreviations: AE, adverse event; EBT, electronic brachytherapy; EBRT, external beam radiation therapy; NA, not available. 
in the combined radiation group were recorded in the first three months following the last radiation treatment.

The one patient with a Grade 3 adverse event (moist desquamation of vaginal mucosa) was treated for recurrent disease in the form of exophytic lesions in the vagina. She had received chemotherapy (two courses of six cycles each) following TAH-BSO for primary endometrial cancer, and subsequently was found to have exophytic lesions in the vagina and left lower pelvis recurrence in October 2008. She was then treated with a high total dose of 80.4 Gy with combined radiation therapy, of which the vaginal brachytherapy dose consisted of five fractions of $5 \mathrm{~Gy}$ each. Two factors may have contributed to the Grade 3 adverse event. First, the 12 cycles of chemotherapy might have lowered the tissue tolerance of the vaginal mucosa, leaving the tissue more susceptible to radiation effects. Second, in standard vaginal brachytherapy, occult cells are treated at a $5 \mathrm{~mm}$ depth and the normally intact superficial mucosa must tolerate the surface dose of vaginal brachytherapy. With the exophytic tumor lesions, the vaginal surface was damaged and not continuously intact while receiving the surface dose equivalent of the 5 Gy prescribed dose to $5 \mathrm{~mm}$ depth. The adverse event resolved completely; however, a combination of prior chemotherapy and superficial lesions at the contact points may predispose such patients to a greater risk of higher-grade adverse events. Dickler et al compared the dosimetry of an EBT source with that of a ${ }^{192}$ iridium source in patients treated for endometrial cancer. The results showed a higher surface dose but generally decreased exposure to nearby tissues with the EBT source. ${ }^{15}$ Dosimetric results from the EBT source may offer certain advantages and disadvantages that must be carefully weighed against those of the ${ }^{192}$ iridium source for each patient. A retrospective study cannot provide a perspective on patient selection for the study intervention because patients who did not receive the treatment are by definition not part of the study. This study is also somewhat limited by the inconsistencies inherent in a multicenter study because treatment planning, delivery, and standards of care vary from site to site. The benefits of a retrospective, multicenter study lie in providing an observation of treatment of a broad patient population in a real world setting.

\section{Conclusion}

This retrospective multicenter study showed that EBT with vaginal cylinders is feasible and well tolerated as a postsurgical adjuvant radiation therapy for primary endometrial cancer. Additional studies are warranted to assess late toxicity and local control further.

\section{Acknowledgment}

The authors wish to thank all site investigators and research staff who supported this study. The authors acknowledge Heike Hausen MD for assistance with medical writing.

\section{Disclosure}

GG has received honoraria within the past two years for speaking at radiation oncology conferences on his experience using electronic brachytherapy as an adjuvant treatment for cancer. Xoft Inc. funded the retrospective data collection study. KW provided statistics support, and Xoft Inc. compensated her time. Xoft Inc. provided funding for this study.

\section{References}

1. Endometrial cancer. National Cancer Institute. Available from: http:// www.cancer.gov/cancertopics/types/endometrial. Accessed 2010 Jun 30 .

2. Lu KH. Management of early-stage endometrial cancer. Semin Oncol. 2009;36:137-144.

3. Lee CM, Szabo A, Shrieve DC, Macdonald OK, Gaffney DK. Frequency and effect of adjuvant radiation therapy among women with stage I endometrial adenocarcinoma. JAMA. 2009;295:389-397.

4. Chadha M, Nanavati PJ, Liu P, Fanning J, Jacobs A. Patterns of failure in endometrial carcinoma stage IB grade 3 and IC patients treated with postoperative vaginal vault brachytherapy. Gynecol Oncol. 1999;75: 103-107.

5. Pearcy R, Petereit D. Post-operative high dose rate brachytherapy in patients with low to intermediate risk endometrial cancer. Radiother Oncol. 2000;56:17-22.

6. Anderson JM, Stea B, Hallum AV, Rogoff E, Childers J. High dose rate postoperative vaginal cuff irradiation alone for stage IB and IC endometrial cancer. Int J Radiat Oncol Biol Phys. 2000;46:417-425.

7. Eltabbakh GH, Piver MS, Hempling RE, Shin KH. Excellent long-term survival and absence of vaginal recurrences in 332 patients with lowrisk stage I endometrial adenocarcinoma treated with hysterectomy and vaginal brachytherapy without formal staging lymph node sampling: Report of a prospective trial. Int J Radiat Oncol Biol Phys. 1997;38: 373-380.

8. Nori D, Merimsky O, Batata M, Caputo T. Postoperative high dose rate intravaginal brachytherapy combined with external irradiation for early stage endometrial cancer: A long term follow-up. Int J Radiat Oncol Biol Phys. 1994;30:831-837.

9. Jolly S, Vargas C, Kumar T, et al. Vaginal brachytherapy alone: An alternative to whole pelvis radiation for early stage endometrial cancer. Gynecol Oncol. 2005;97:887-892.

10. Solhjem MC, Petersen IA, Haddock MG. Vaginal brachytherapy alone is sufficient adjuvant treatment for patients with surgical stage I endometrial cancer. Int J Radiat Oncol Biol Phys. 2005;62:1379-1384.

11. Small W Jr, Zeytinoglu M, Keh R, et al. Endometrial adenocarcinoma invasive to $1 / 2$ the myometrial thickness: Analysis of prognostic variables for recurrence and survival. Int J Radiat Oncol Biol Phys. 2001; 51 Supp1 2:S35-S36.

12. Petereit DG, Tannehill SP, Grosen EA, Hartenbach EM, Schink JC. Outpatient vaginal cuff brachytherapy for endometrial cancer. Int J Gynecol Cancer. 1999;9:456-462.

13. McCloskey SA, Tchabo NE, Malhotra HK, et al. Adjuvant vaginal brachytherapy alone for high risk localized endometrial cancer as defined by the three major randomized trials of adjuvant pelvic radiation. Gynecol Oncol. 2010;116:404-407. 
14. Mehta VK, Algan O, Griem KL, et al. Experience with an electronic brachytherapy technique for intracavitary accelerated partial breast irradiation. Am J Clin Oncol. 2010;33:327-335.

15. Dickler A, Kirk MC, Coon A, et al. A dosimetric comparison of Xoft Axxent Electronic Brachytherapy and iridium-192 high-dose-rate brachytherapy in the treatment of endometrial cancer. Brachytherapy. 2008; 7:351-354.

16. Small W, Erickson B, Kwakwa F. American Brachytherapy Society survey regarding practice patterns of postoperative irradiation for endometrial cancer: Current status of vaginal brachytherapy. Int J Radiat Oncol Biol Phys. 2005;63:1502-1507.
17. Nout RA, Putter H, Jürgenliemk-Schulz IM, et al. Quality of life after pelvic radiotherapy or vaginal brachytherapy for endometrial cancer: First results of the randomized PORTEC-2 trial. J Clin Oncol. 2009;27: 3547-3556.

18. Dickler A, Puthawala MY, Thropay JP, Bhatnagar A, Schreiber G. Prospective multi-center trial utilizing electronic brachytherapy for the treatment of endometrial cancer. Radiat Oncol. 2010;20:67.

\section{Publish your work in this journal}

OncoTargets and Therapy is an international, peer-reviewed, open access journal focusing on the pathological basis of all cancers, potential targets for therapy and treatment protocols employed to improve the management of cancer patients. The journal also focuses on the impact of management programs and new therapeutic agents and protocols on

\section{Dovepress}

patient perspectives such as quality of life, adherence and satisfaction. The manuscript management system is completely online and includes a very quick and fair peer-review system, which is all easy to use. Visit http://www.dovepress.com/testimonials.php to read real quotes from published authors.

Submit your manuscript here: http://www.dovepress.com/oncotargets-and-therapy-journal 